\title{
A complexidade da obesidade e o processo de viver após a cirurgia bariátrica: uma questão de saúde coletiva
}

\author{
The complexity of obesity and life after bariatric surgery: \\ a public health issue
}

Liete Francisco Marcelino ${ }^{1}$

Zuleica Maria Patrício ${ }^{2}$

\footnotetext{
${ }^{1}$ Universidade do Sul de Santa Catarina (UNISUL), Campus Tubarão. Avenida José Acácio Moreira 787, Bairro Dehon. 88704-900 Tubarão SC.

${ }^{2}$ Universidade Federal de Santa Catarina. zucamp@hotmail.com.
}

\begin{abstract}
The high incidence of obese people submitted to bariatric surgery as treatment for obesity has prompted the need to carry out a survey to ascertain the repercussions of this treatment on the health of a group of individuals subjected to such surgery. Data were collected via in-depth interviews, focusing on identification of the background to the situation that led to the decision to submit to obesity surgery and the quality of daily life after treatment. The analysis showed that the constant difficulties and frustrations in combating obesity and the expectation of the definitive cure led to the decision to submit to surgery in that group. However, life after surgery was characterized as a continuous process of challenges with further hurdles in order to learn how to live again on a day-to-day basis and return to full health. This also presents challenges for the health team as it involves greater investment in public health, improvement strategies for prevention and control of obesity of the population and comprehensive care actions for those individuals. Such complexity requires interdisciplinary expertise with an approach in line with the emotional and socio-cultural issues of this population group.
\end{abstract} Key words Obesity, Bariatric surgery, Health Care, Health promotion, Public health
Resumo O conhecimento dos altos indices de pessoas obesas submetidas à cirurgia bariátrica como tratamento da obesidade, gerou a necessidade de realizar um estudo com o objetivo de conhecer as repercussões desse tratamento na saúde de um grupo de indivíduos a ele submetidos. Os dados foram coletados por entrevista em profundidade, com foco na identificação da história da situação de obesidade que gerou a decisão pela cirurgia e o processo de viver o cotidiano após esse tratamento. A análise mostrou que as constantes dificuldades e frustrações no combate da obesidade e a expectativa da cura definitiva geraram, naquele grupo, a decisão de se submeter à cirurgia. Entretanto, a vida após esse tratamento, ainda se caracterizava como um processo contínuo de enfrentamentos, com mais desafios para reaprender a viver o cotidiano e resgatar a saúde integral. Esse contexto implica, também, desafios para a equipe de saúde; envolve maior investimento na saúde coletiva, aperfeiçoamento nas estratégias de prevenção e controle de obesidade da população e também ações de cuidado integral àqueles indivíduos. Tal complexidade exige atuação interdisciplinar com olhar sincronizado com as questões afetivas e socioculturais dessa população. Palavras-chave Obesidade, Cirurgia bariátrica, Cuidados de saúde, Promoção da saúde, Saúde coletiva 


\section{Introdução}

No começo deste século, a Organização Mundial da Saúde $(\mathrm{OMS})^{1}$ chamava atenção para o fato de que no mundo há mais de um bilhão de adultos com sobrepeso e aproximadamente trezentos milhões de adultos obesos, sendo que cerca de $60 \%$ da população mundial, num futuro próximo, apresentarão algum problema de saúde relacionado à obesidade.

Ao final da primeira década, os resultados de pesquisas no mundo, tornaram a obesidade, segundo a $\mathrm{OMS}^{1}$, uma doença epidêmica global do século 21 que precisa ser encarada mais efetivamente tendo em vista o alto índice na população infantil e as evidentes consequências na saúde integral da pessoa obesa. A condição obesidade favorece em grande escala o risco de aparecimento e agravamento de doenças crônicas e de sofrimentos psicosociais.

No mundo inteiro há mobilizações científicas e políticas focadas para esse problema. De tal ordem que, em fevereiro de 2011, com a presença da direção da OMS, ministros da saúde de países do continente americano, reunidos no México durante a Consulta Regional de Alto Nível das Américas Contra as Enfermidades Crônicas não Transmissíveis (ECNT), assinaram uma declaração propondo a criação de políticas públicas com o fim de combater a obesidade nas respectivas nações. Tal como prescrito na Carta Europeia de Luta contra a Obesidade, essa declaração reconhece a liderança da área da saúde nessa luta contra a doença e seus agravos, mas coloca ênfase no caráter intersetorial das ações, propondo o engajamento dos setores de educação, cultura, comércio e mídiaª

A epidemia da obesidade na Europa é considerada um dos maiores desafios para a saúde pública, tendo em vista a sua origem na mudança rápida de determinantes sociais, econômicos e ambientais do estilo de vida das pessoas ${ }^{3}$. No Brasil, segundo o Ministério da Saúde, a parcela da população cujo Índice de Massa Corporal (IMC) é igual ou superior a $30-$ consideradas obesas - passou de $11,4 \%$ para $13,9 \%$ em apenas três anos, entre 2006 e 2009. E a tendência é aumentar. Estima-se ainda que 4 milhões de brasileiros tenham atingido o estágio de obesidade mórbida, quando o IMC chega a $40^{4}$.

A OMS classifica uma situação de obesidade quando o IMC (peso em $\mathrm{kg}$ dividido pelo quadrado da altura em metro) encontra-se acima de $30 \mathrm{~kg} / \mathrm{m}^{2}$. Quanto à gravidade, a OMS propõe a seguinte classificação: obesidade grau I, quando o IMC está entre 30 e $34,9 \mathrm{~kg} / \mathrm{m}^{2}$; obesidade grau II, quando o IMC está entre 35 e $39,9 \mathrm{~kg} / \mathrm{m}^{2}$; e obesidade grau III, quando o IMC ultrapassa $40 \mathrm{~kg} / \mathrm{m}^{2}$.

O crescimento do número de pessoas obesas está sendo considerado um problema de saúde pública, principalmente porque esses casos estão sobrecarregando o sistema público de saúde no tratamento de excesso de peso e doenças ligadas a esse problema. A questão é que a obesidade é uma doença multifatorial, relacionada com diversos aspectos, desde aqueles de ordem genética até os de ordem socioambiental, podendo-se depreender que $95 \%$ ou mais dos casos estão intimamente ligados ao estilo de vida ${ }^{5}$.

A obesidade mórbida ou severa é acompanhada de uma série de complicações que comprometem a saúde de maneira geral e põe em risco a vida do indivíduo. Entre as complicações estão os distúrbios cardiovasculares, ortopédicos, digestivos, endócrinos, dermatológicos e respiratórios ${ }^{6,7}$.

Os programas de atenção à saúde têm apresentado estratégias de promoção que buscam estimular o interesse das pessoas por práticas saudáveis, bem como tem havido incremento no tratamento das pessoas que sofrem de obesidade mórbida, prevenindo-se diversos agravos e suas complicações. Apesar das várias intervenções para combater a obesidade, há também um aumento da sua gravidade, em razão da utilização de métodos inadequados por parte das pessoas obesas. Estas, além de comprometerem o metabolismo, são normalmente ineficazes e facilitam a recuperação do peso perdido, tornando o tratamento ainda mais difícil ${ }^{8}$.

A questão é a complexidade que envolve a situação de ser obeso. Por ser a obesidade um fenômeno bastante centrado no aspecto comportamental, a meta de atingir um peso saudável passou a exigir mudanças nos hábitos alimentares, na prática do condicionamento físico e na atenção à saúde psíquica. Essa meta, para o obeso mórbido, é ainda mais difícil, muitas vezes até "impossível", devido à gravidade do seu tipo de obesidade, que exige medidas mais drásticas de tratamento.

Atualmente, existem diversos tratamentos para a perda de peso, dentre os quais se destaca a variedade de dietas, psicoterapias, medicamentos e programas de atividades físicas. Porém, a maioria dos obesos mórbidos não obtém sucesso com esses recursos. Com isso surgem outros problemas, além daqueles associados ao ganho de peso, como, por exemplo, a frustração e a 
ansiedade constantes, o estresse e a depressão, que influenciam ainda mais no comportamento alimentar inadequado e no agravamento da morbidade 9 .

A avaliação do cenário da obesidade na população, e das dificuldades de as pessoas seguirem o tratamento habitual e terem sucesso com ele, estimulou a ciência médica a desenvolver um tratamento via intervenção cirúrgica.

Desde a década de setenta do século passado, as pessoas que sofrem de obesidade mórbida podem contar com outro tipo de tratamento: a cirurgia bariátrica. Apesar de terem sido desenvolvidos poucos estudos com a população que se submeteu a este tratamento, especialmente de caráter qualitativo, segundo a avaliação de médicos que atendem essa população, a cirurgia tem se mostrado um tratamento que traz novas perspectivas para os indivíduos obesos.

A cirurgia bariátrica, também conhecida como gastroplastia e cirurgia de redução de estômago, é uma opção para pessoas com obesidade mórbida que não conseguem perder peso pelos métodos tradicionais ou para quem sofre de problemas crônicos de saúde relacionados a essa doença9.

A cirúrgica bariátrica também tem sido utilizada para tratamento de pessoas obesas que são portadoras de Diabetes Mellitus tipo II, pois, ao induzir significante perda ponderal em indivíduos obesos, diminui a resistência à insulina e, consequentemente, os fatores de risco cardiovasculares ${ }^{10}$.

O procedimento possibilita a redução do peso inicial, que pode ser em média 40\% em um ano a partir da realização da cirurgia. A intervenção reduz o estômago em cerca de 20 centímetros cúbicos, ou seja, o estômago reduzido perde até $90 \%$ de sua capacidade de absorção $0^{5-7}$.

Devido a essa redução, diminui a capacidade do estômago de suportar a quantidade habitual de alimentos. Com isso, o estômago enche-se rapidamente; a mensagem de saciedade é transmitida ao cérebro fazendo com que a pessoa coma bem menos do que antes.

As indicações para a cirurgia como tratamento da obesidade mórbida baseiam-se em diversos fatores: IMC acima de $40 \mathrm{~kg} / \mathrm{m}^{2}$, ou acima de $35 \mathrm{~kg} / \mathrm{m}^{2}$ na presença de doenças associadas; fracasso de métodos tradicionais de emagrecimento; ausência de causas endócrinas de obesidade, como síndrome de Cushing; e avaliação favorável das possibilidades psíquicas de o indivíduo suportar as transformações radicais de comportamento impostas pela cirurgia ${ }^{6}$.
Foi aprovada no Brasil em 17 de fevereiro de 2011, uma nova regulamentação que autoriza a utilização da banda gástrica no tratamento da obesidade moderada, de maneira que indivíduos com o índice de massa corpórea (IMC) a partir de $30 \mathrm{~kg} / \mathrm{m}^{2}$ e que sofram de alguma doença associada à obesidade, como diabetes, hipertensão arterial e apneia do sono, estão aptos para o tratamento cirúrgico ${ }^{11}$.

A literatura destaca que procedimentos drásticos só devem ser adotados quando a obesidade estiver ameaçando a vida e os tratamentos convencionais não fizerem efeito. A indicação da cirurgia somente será adotada após minuciosa análise dos múltiplos aspectos clínicos da pessoa obesa, o que exige atenção de uma equipe multidisciplinar, e somente após ela demonstrar estar esclarecida a respeito de todos os detalhes, referentes ao seu diagnóstico, aos benefícios e riscos da cirurgia, bem como às repercussões e necessidades de tratamento posterior ${ }^{5,7,12}$. Sem dúvida, se o "Termo de Consentimento Informado", prescrito pela Sociedade Brasileira de Cirurgia Bariátrica e Metabólica (SBCBM) ${ }^{13}$, for aplicado com princípios éticos, somente assinará o Termo para se submeter à cirurgia aquela pessoa obesa com extrema necessidade de tratamento cirúrgico.

Os resultados esperados com a cirurgia bariátrica incluem perda de peso, melhora das comorbidades relacionadas e aperfeiçoamento da qualidade de vida em geral. Estudos clínicos revelam que, após a cirurgia, muitos indivíduos perdem peso rapidamente e continuam a perder até 18 a 24 meses após o procedimento, podendo manter 50 a $60 \%$ da perda do excesso de peso de 10 a 14 anos após a cirurgia ${ }^{5,7}$.

Quanto às repercussões não satisfatórias, a princípio, estão as intercorrências pelo próprio ato cirúrgico, como qualquer procedimento invasivo com anestesia geral, o risco de infecção e a possibilidade, mesmo que remota, de embolia pulmonar. Pode haver, também, complicações decorrentes do excesso de peso ou por agravamento de doenças associadas, como cardiovasculares e diabetes. O risco total de complicações varia entre 10 e $15 \%$ e a mortalidade está em torno de 0,3 a $1,6 \%$, sendo mais frequente em indivíduos com muitos fatores de risco associados à obesidade ${ }^{7}$.

A atenção aos riscos cirúrgicos e ao tratamento posterior muito prolongado torna-se mais importante frente à evidência do alto número de gastroplastias no contexto brasileiro. E, a tendência é aumentar nos próximos anos, em função da divulgação dos casos de sucesso e da diminuição 
dos riscos cirúrgicos e, também, pelo fato desse tratamento estar inserido em planos particulares de saúde e no Sistema Único de Saúde (SUS).

No Brasil, a cirurgia bariátrica começou a ser realizada em maior escala a partir do ano 2000 , quando alguns planos de saúde particulares e a rede pública passaram a pagar os custos do procedimento. Esse tratamento foi regulamentado no SUS pela Portaria no 628/GM, de 26 de abril de 2001, cujo protocolo foi aperfeiçoado em $2005^{14}$. Desde então, o usuário do SUS com obesidade mórbida passou a realizar essa cirurgia em alguns centros do País. A procura por estes centros tem sido muito grande e a demanda do serviço público ainda não consegue ser atendida como esperado, gerando longas filas de espera ${ }^{15}$.

Dados da SBCBM apontam que em 2010 foram realizadas 64,04 mil cirurgias no país, alta de $275 \%$ em relação a 2003, ano em que os primeiros registros foram coletados, e de $33 \%$ em relação a 2009. Pelo SUS, o número de cirurgias aumentou 23,7\% entre 2007 e 2009, chegando a 3.681 intervenções, mesmo com filas de espera de até oito anos. Em hospitais vinculados ao SUS o número de cirurgias aumentou quase $800 \%$ entre 2001 e 2010. Em unidades particulares, o crescimento registrado na última década é menor, cerca de $300 \%$. "Os números fazem do Brasil o segundo colocado no ranking de cirurgias bariátricas, atrás apenas dos Estados Unidos, com 300 mil procedimentos em 2010"13.

Chama-se atenção que construir uma vida mais saudável - resultado esperado pela população que se submete a cirurgia - é um processo muito complexo, pois envolve a interligação dos aspectos físicos, psíquicos e sociais, e varia de indivíduo para indivíduo. Nesse sentido, existe a recomendação do Ministério da Saúde para que haja um acompanhamento multiprofissional e sistemático aos indivíduos que se submeteram ao tratamento cirúrgico. No texto do "Termo de Consentimento Informado", prescrito pela SBCBM para ser aplicado junto ao candidato à cirurgia, fica evidente essa necessidade: "A realização correta e eficaz da intervenção cirúrgica indicada não impede futuro ganho de peso se não houver o devido acompanhamento e disciplina no tratamento"13.

No cotidiano das práticas profissionais em serviços de saúde da rede pública, é comum encontrar-se situações que evidenciam limitações das equipes de saúde na atenção específica aos indivíduos submetidos à cirurgia bariátrica, geralmente por desconhecimento da complexidade dos cuidados requeridos por essa população e, espe- cialmente, pela dificuldade de compreender o caráter subjetivo do enfrentamento das pessoas no processo de readaptação ao novo estilo de vida.

Esses aspectos, juntamente com a constatação da carência de estudos qualitativos sobre o processo de viver e a saúde integral das pessoas submetidas a esse tratamento, motivaram a realização de uma pesquisa que teve como objetivo conhecer a repercussão da cirurgia bariátrica na saúde de um grupo de indivíduos obesos submetidos a este tipo de tratamento.

\section{Método}

O estudo foi desenvolvido com abordagem qualitativa tendo em vista o caráter de seu objetivo e a situação ímpar de cada pessoa que se submete à cirurgia bariátrica. Em virtude da complexidade desse tipo de processo, há necessidade de abordar a vivência dos sujeitos na sua história particular, considerando-os na condição de pessoas obesas e como integrantes de uma população que já tentou vários tipos de tratamento e agora vive o desafio de retomar a condição de ser obesa com outro sentido.

O processo de levantamento e análise dos dados seguiu a orientação de pesquisas do tipo "estudo de caso". Indo além do caráter exploratório, esse processo possibilitou a compreensão do fenômeno na subjetividade da vida particular de cada sujeito, bem como mostrou o caráter coletivo desse fenômeno, em seu universo de significados e aspirações comuns.

Participaram do estudo 6 sujeitos, na faixa etária de 24 a 47 anos, residentes no município de Tubarão-SC, com as seguintes características: 5 do sexo feminino e 1 do sexo masculino; 3 solteiros, 1 casado e 2 divorciados; todos trabalhadores, sendo 3 professores, 1 técnico de enfermagem, 1 advogado e 1 comerciante, que também cursava o $3^{\circ}$ grau.

Foram definidos os seguintes critérios de inclusão: ter se submetido à cirurgia bariátrica com a finalidade de tratamento da obesidade mórbida; estar em processo de adaptação à nova situação; demonstrar interesse e disponibilidade de tempo para responder as questões em profundidade e assinar o Termo de Consentimento Livre e Esclarecido. O projeto foi aprovado pelo Comitê de Ética em Pesquisa da Universidade do Sul de Santa Catarina.

Para o levantamento dos dados foi utilizada a técnica da entrevista em profundidade, com apoio de um formulário composto por questões aber- 
tas dirigidas à identificação da história de obesidade dos sujeitos e aos significados relativos ao processo posterior à realização da cirurgia.

A análise dos dados, seguindo o modelo qualitativo adotado, centrou-se no levantamento de categorias $^{16}$ e, posteriormente, na leitura reflexiva do conjunto destas categorias para identificação de temas emergentes ${ }^{17}$. Esta técnica fez emergir dois temas centrais, a saber: "A dificuldade de lidar com a complexidade da obesidade gerando a opção pela cirurgia" e "Desafios no processo pós-cirúrgico para reaprender a viver o cotidiano e resgatar a saúde integral”.

\section{Apresentação e discussão dos resultados}

\section{A dificuldade de lidar com a complexidade da obesidade gerando a opção pela cirurgia bariátrica}

O evento obesidade na vida das pessoas que participaram do estudo caracterizava-se como um fenômeno de perdas, desconfortos e desafios. O processo de viver era uma constante condição de riscos para doenças e seus agravos e de insatisfações cotidianas, envoltas pela luta contínua para adotar hábitos mais sadios que pudessem tratar de vez o problema.

Esses aspectos foram evidenciados não apenas no conteúdo dos depoimentos, mas também na linguagem não verbal, manifestada por expressões faciais que demonstravam desconforto e incômodo com a condição de ser obeso.

A convivência com a obesidade, no contexto deste estudo, manifesta-se vinculada de forma significativa a comorbidades e a outras condições existenciais que limitam o processo de viver saudável.

Os problemas físicos identificados, associados à obesidade, foram: hipertensão arterial, orteoartralgia, irregularidade menstrual, ovário policístico, dificuldade para engravidar, diabetes Mellitus e dislipidemias.

Revelou-se também que a falta de flexibilidade das articulações, a dor e o edema associados às osteoartrites se intensificaram com a obesidade. O peso contribuiu para a sobrecarga articular comprometendo os movimentos e limitando os sujeitos na execução de suas atividades físicas cotidianas.

Decorrente das comorbidades e de suas reações incapacitantes, estavam o afastamento do trabalho, o ônus econômico pelo custo do tratamento e as restrições para lazer e atividades físicas, in- cluindo-se também o ônus emocional devido a preconceitos em relação à aparência corporal.

Ficou evidente que a obesidade também interfere na vivência da sexualidade e na função sexual. A perda da libido e a disfunção hormonal ocasionaram danos significativos para a saúde dos sujeitos. Por outro lado, o medo da não aceitação do outro, devido ao seu corpo "anormal", faz com que o obeso crie restrições nos relacionamentos afetivos e sexuais. A baixa autoestima, gerada pelo autopreconceito, é o principal desencadeador dos problemas relacionados à afetividade e à intimidade nos relacionamentos.

Outro aspecto evidenciado, também apontado em outras pesquisas, diz respeito ao desequilíbrio entre o dispêndio reduzido de energia devido à falta de atividade física e a adoção de dietas altamente energéticas. Esse é um dos principais fatores determinantes da obesidade, constituindo-se numa das preocupações das políticas públicas de saúde.

Todos os sujeitos possuíam práticas alimentares inapropriadas, na quantidade e na qualidade dos alimentos e na dinâmica cotidiana de se alimentar. Um dos motivos para a dificuldade de manter o controle alimentar estava no fato de o prazer de comer tornar-se um substituto no atendimento de outro tipo de necessidade. Inúmeros artigos sobre essa temática, na área da psicologia, chamam atenção para o fato de a pessoa acima do peso ter problemas de aceitação, seja pelo preconceito social, seja pela dificuldade de adaptação em relação ao vestuário e a certos espaços físicos.

Diante desse contexto é importante refletir sobre o fato de que o obeso vivencia problemas de ordem psicossocial que emergem como resultado da obesidade e que, de certa forma, acabam potencializando fatores que predispõe a pessoa à manutenção ou ao agravamento da doença.

Muitos dos sofrimentos psicológicos da pessoa portadora de obesidade são decorrentes dos estigmas sociais em relação a essa situação. Sem dúvida, as consequências psicossociais da obesidade também derivam de valores ligados à cultura atual que considera o corpo gordo feio e inaceitável $^{18,19}$.

No estudo, evidenciou-se que os sentimentos que levam à autodepreciação e à baixa autoestima, estavam relacionados com a ênfase midiática à inadequação do obeso aos padrões de beleza estabelecidos pela sociedade atual, acrescida da representação da pessoa obesa como não saudável.

Identificou-se que a falta de atividade física era uma constante no cotidiano de vida dos su- 
jeitos, fato que potencializou o evento obesidade. Apesar da prescrição médica para essa conduta, havia impedimentos existenciais para essa prática, em razão do risco de depreciações e julgamentos negativos que pudessem sofrer nos ambientes dessas atividades. A preocupação com a exposição da sua imagem corporal preponderava em detrimento aos possíveis benefícios da atividade física na melhoria de sua condição.

A carência de autoestima, fortemente evidenciada nos sujeitos, é justificada por eles pelo preconceito e pela discriminação existente na sociedade, fato este observado nas mais variadas e corriqueiras situações, como brincadeiras maliciosas em programas de televisão e textos publicados. Argumentam que a cultura da exclusão de "corpos" que não sejam magros já está explícita quando as pessoas obesas têm dificuldades para encontrar roupas para o seu tamanho ou mesmo para conseguir um emprego.

A percepção de estarem sendo rejeitados gerou estados de reclusão e também de relações pessoais conturbadas, o que provocou perdas afetivas significativas, e consequentes estados de tristeza, ansiedade e até de depressão.

Desta forma, o problema foi sendo reforçado: mais ganho de peso, mais problemas de saúde, mais sentimento de discriminação e menos possibilidades pessoais de promover uma vida mais saudável. Esse constante estado de insatisfação impulsionou todos os sujeitos para tentativas drásticas de redução de peso, algumas destas consideradas de risco.

A utilização de estratégias extremas acarretou sérios prejuízos aos sujeitos, especialmente por inadequações no atendimento aos critérios de utilização. Essa situação foi fortalecida pela falta de um acompanhamento profissional mais centrado na pessoa, mais holístico, mais multidimensional, para além da atenção exclusiva aos seus sinais e sintomas imediatos.

A decisão que o sujeito tomou para se submeter à cirurgia bariátrica aconteceu somente após muita reflexão sobre as constantes insatisfações e ao grande cansaço e desânimo em relação aos inúmeros tratamentos que fez sem sucesso.

\section{O processo pós-cirúrgico como desafio para reaprender a viver o cotidiano e promover a saúde integral: a necessidade de cuidados integrais}

Para a população do estudo, o processo de viver o período pós-operatório mediato, representado, especialmente, pela grande redução de peso, caracterizou-se como o início do maior desafio do processo. Para alcançar a meta defini$\mathrm{da}$ os indivíduos tiveram que considerar a obrigatoriedade de mudar seus hábitos relacionados à alimentação e às atividades físicas.

Poucos meses após a cirurgia, com a perda substancial de peso, os indivíduos puderam atingir uma melhora significativa nas comorbidades, como nos casos de artralgias, disfunção hormonal, taxas de glicose alteradas e hipertensão. Também foi positivo poder retomar certas práticas, incluindo atividades de trabalho e encontros sociais. Tudo isso, segundo eles, refletiu consideravelmente na sua qualidade de vida e na qualidade de vida das demais pessoas com as quais conviviam. A repercussão principal foi na autoestima, pela satisfação consigo próprio, por ter realizado a cirurgia e perceber a contínua perda de peso.

Com todas essas repercussões houve influência positiva nas suas relações sociais. O estímulo proporcionado pela perda de peso e consequente aumento da autoestima pôde ajudar o sujeito desmotivado, ou desadaptado, a procurar formas de resgatar o entusiasmo e a vontade de viver, principalmente em atividades de grupo.

Mas, para essa população chegar nesse ponto, precisou desenvolver uma grande capacidade de superação, frente às inúmeras dificuldades do pós-operatório, e muita força de vontade para "reconstruir-se" como pessoa dia após dia. $\mathrm{Na}$ época do estudo alguns sujeitos ainda não tinham conseguido atender essa expectativa em sua plenitude. A análise dos dados mostra que algum tipo de ameaça, perda ou desafios da vida particular, se mantinham presentes na luta contra a obesidade.

O período imediato após a cirurgia confirmou ser o mais difícil para o operado. É a fase de maior desconforto e de várias tentativas de adaptação à nova dieta. Associado a tudo isso ainda havia muita expectativa, ansiedade e insegurança em relação à nova situação.

O tipo e a quantidade da alimentação orientada para o pós-operatório variaram na população do estudo. Segundo a literatura, os procedimentos dietéticos dependem do método cirúrgico adotado ${ }^{6}$.

A necessidade de cuidados foi mais acentuada no processo de readaptação alimentar. Esta fase obriga o operado a grandes sacrifícios, exigindo dele muita força de vontade, muito autocontrole e determinação.

A dificuldade e o desconforto mais acentuados ocorreram na fase da ingestão de alimenta- 
ção líquida. Nesse período o indivíduo encontra-se fragilizado fisicamente, pelas reações comuns à cirurgia, e também psicologicamente, em razão da privação alimentar e dos sentimentos de incertezas.

O início da fase de adaptação física e mental à nova maneira de se alimentar, agregada ao estado de debilidade, imprimiu um caráter bastante limitante na qualidade de vida dos sujeitos. O vômito foi o problema mais referido. Em alguns casos, essa situação persistiu durante meses.

Outro mal apresentado esteve relacionado à síndrome do Dumping, que é uma consequência do rápido esvaziamento das substâncias do estômago para o intestino delgado. O estômago não quebra todas as enzimas do açúcar e este acaba chegando "bruto" ao intestino. Algumas vezes, este mecanismo é ativado quando são consumidas grandes quantidades de açúcar ou outros tipos de alimentos. Em geral, os resultados são extremamente desagradáveis, podendo provocar náusea, fraqueza, transpiração excessiva, desmaio e, em alguns casos, diarreia e alopécia ${ }^{5,6,20,21}$.

No grupo estudado, mesmo após meses do tratamento, ainda havia dificuldades no controle da prática alimentar. Alguns sujeitos apresentavam intolerância maior e muita insegurança na ingestão de certos tipos de alimentos. Houve casos em que a persistência desses problemas ocasionou desequilíbrio de nutrientes importantes.

A cirurgia bariátrica possibilita erradicar as comorbidades inerentes à obesidade, assim como promove a redução IMC, porém tende a provocar déficits nutricionais importantes, se não houver suplementação nutricional adequada ${ }^{6,7}$. Essa situação exige acompanhamento profissional sistemático com apoio em exames laboratoriais. A alopécia é exemplo disso, pois pode estar relacionada à deficiência de zinco, de proteínas e de ácidos graxos essenciais ${ }^{5,6}$.

Todos os sujeitos criaram limitações psicossociais significativas em decorrência da insegurança e dos receios quanto aos resultados da cirurgia, o que provocou exacerbação de algumas características de seu estado anterior. A questão é ainda a compulsão por comida. Com a cirurgia o indivíduo se sente impedido de comer como antes, mas continua compulsivo - o que faz com que desenvolva outros distúrbios afins, como forma de compensação.

Assim como relata a literatura, este estudo também evidenciou que uma parcela de ex-obesos mórbidos, depois de emagrecer com a cirurgia bariátrica desenvolve depressão, bulimia, anorexia, dependência de álcool e outras drogas, compulsões por jogos, compras ou sexo. Um dos motivos seria porque a redução do estômago não permite que eles descontem mais nos alimentos as carências afetivas, entre outras razões inconscientes que os levaram a engordar. Por isso, é comum procurarem novas válvulas de escape ${ }^{5}$.

Nesse processo de adaptação, houve a necessidade de mudança de rotinas, o que provocou algumas dificuldades nas relações sociais, em especial na família, principalmente em decorrência do assédio à comida e o conflito com suas limitações alimentares. Esse processo exigiu dos indivíduos uma maior reflexão sobre si mesmos, o que provocou o emergir de diferentes significados ou o reforço de questões existenciais.

Houve recorrência da baixa autoestima em relação á imagem corporal, agora atribuída aos resultados provenientes da perda ponderal acelerada, a qual confere ao corpo grandes excessos de pele na forma de flacidez localizada. Confirmando a força do fator estético que dirigiu essa população para se submeter à cirurgia, a insatisfação com a nova aparência física gerou a necessidade de corrigir as "imperfeições" por meio da cirurgia plástica.

Esses aspectos corroboram o estudo de Carvalho e luz ${ }^{22}$, especialmente ao citarem o trabalho de Spink e Medrado publicado em 2004, que aponta para o importante acervo de possibilidades que a pessoa dispõe para a vida futura advindo dos significados e dos sentidos incorporados ao longo de sua história, e o papel do corpo, como o principal espaço para sua construção, representando uma força poderosa e inevitável na vida em sociedade.

Por conta de toda a situação construída após a cirurgia bariátrica, a necessidade de acompanhamento sistemático de profissionais para o monitoramento da saúde em todas as suas dimensões, torna-se uma constante na vida dos sujeitos. Esse aspecto é tão mais importante quando são consideradas também as sérias complicações que podem ocorrer no pós-operatório, incluindo o risco eminente de morte, seja por transtornos físicos ou psíquicos. A atenção precisa ser ainda mais acentuada frente à sutileza de problemas que possam impulsionar o sujeito para o suicídio, que, conforme a literatura, não é incomum na situação pós-cirúrgica ${ }^{21,23,24}$.

Embora a atenção a esses problemas conste no protocolo estabelecido para a atenção às pessoas que se submetem a essa cirurgia, o estudo mostrou que há dificuldade para encontrar profissionais capacitados fora dos centros de referência para atender a complexidade dos proble- 
mas de saúde dessas pessoas. Isso reforçou, nos sujeitos, sentimentos de insegurança quanto à manutenção do tratamento pós-cirúrgico e a realização plena de suas expectativas.

O apoio emocional foi tido como componente prioritário no tratamento, mas para além do cuidado individual e específico. Por ser uma nova situação que costuma repercutir na família e nos demais grupos de convívio íntimo da pessoa obesa, incluindo aqueles de trabalho, a preocupação com a qualidade das relações sociais torna-se ainda mais importante no processo de desenvolvimento de estratégias de enfrentamentos para uma convivência coletiva mais saudável e que contribua para o sucesso do tratamento.

\section{Síntese final e recomendações}

O número de casos de obesidade cresce progressivamente, constituindo-se num sério problema de saúde pública, especialmente, em razão da complexidade da situação "ser obeso" constituirse em um fenômeno de difícil manejo.

Essa situação está provocando um movimento mundial diferenciado, também pela negação da prática cirúrgica como tratamento generalizado para obesidade mórbida. Percebe-se o desafio que as políticas públicas de saúde estão focando de maneira mais contundente: a prevenção da obesidade, de tal forma que diminua, sensivelmente, $o$ número de pessoas que necessitem da cirurgia bariátrica como solução para seus problemas.

Esse dado é relevante, diante da expectativa de crescimento do número de pessoas que se decidirá por este tipo de tratamento, nesta segunda década do século 21, em razão dos fatores apontados na introdução.

Tal como descrito em outros trabalhos sobre essa temática, a população do presente estudo também, em decorrência dos inúmeros fracassos no tratamento da obesidade e diante da possibilidade de realizar a cirurgia bariátrica, colocou todas as suas esperanças nesse procedimento, visualizando a "cura definitiva" para a obesidade e a consequente resolução dos problemas de saúde associados.

Entretanto, a imagem mental que fica ao final da análise dos dados sobre a descrição de cada vida estudada, representa um conjunto particular de situações complexas de âmbito multidimensional, que muito se aproxima de um fenômeno paradoxal.

A decisão pelo tratamento cirúrgico no grupo estudado, a princípio se caracterizou como a alternativa final no processo de busca para a cura da obesidade, na expectativa de um viver com menor desconforto e maior insatisfação com seu corpo. Na avaliação dos sujeitos, a cirurgia, apesar de todas as dificuldades no começo, pelo fato de facilitar a redução de peso de imediato, promoveu condições para melhorar aspectos físicos, emocionais e sociais.

Entretanto, em termos de saúde integral, não se pode avaliar o resultado da cirurgia somente pela perda de peso. A análise do processo que envolve o desafio de o sujeito ter o livre arbítrio para construir a qualidade da sua vida cotidiana em sua plenitude e poder tornar realidade o desaparecimento das doenças associadas, precisam ser considerados na avaliação do resultado da cirurgia. Há casos em que o indivíduo operado continua convivendo - e sofrendo - com fatores que o levaram à obesidade e às suas consequências.

A análise minuciosa e reflexiva dos depoimentos e da linguagem não verbal deixou mais evidente que a pessoa, mesmo após meses da cirurgia, continua vivendo a situação de ser obesa e que tratar a obesidade ainda é uma "batalha" cotidiana. Essa luta, dependendo, em grau, do suporte socioeconômico que a pessoa possui, acaba repercutindo em sua saúde integral, especialmente nas dimensões psicossociais.

Considera-se, com base na literatura e nos dados empíricos desse estudo, que a cirurgia bariátrica possa ser o tratamento definitivo da obesidade apenas para alguns tipos de pessoas obesas. A imagem de que esse procedimento possa "milagrosamente" transformar as pessoas com obesidade mórbida em pessoas sadias, precisa ser evitada, pois a maioria das pessoas que se submete a esse tratamento continua convivendo com os fatores limitantes de sua condição histórica.

Em virtude dessas considerações, entende-se que os critérios estabelecidos devam ser rigorosamente considerados na indicação do tratamento cirúrgico, a ponto de garantir que essa seja a última alternativa de intervenção.

Para tanto, a consulta com o cirurgião não basta. É imprescindível que haja atenção multiprofissional nas fases pré e pós-cirúrgica, visando ações de cuidado integral que possam atender as expectativas do indivíduo com relação a mudanças estéticas de seu corpo, suas limitações e possíveis consequências do tratamento cirúrgico, tendo em vista sua situação particular e seu contexto.

O resultado da cirurgia é mensurado pelo sucesso do próprio ato cirúrgico sem complicações, e quando há redução acentuada do peso, e 
pela incorporação, pelo operado, de hábitos saudáveis na vida cotidiana, sem sofrimentos limitantes. O resultado é assegurado quando há remissão ou melhora das afecções associadas (hipertensão, diabetes, dislipidemia, problemas ortopédicos e apneia do sono, entre outras) e sensíveis incrementos na qualidade de vida, especialmente advindos da resignificação de valores sobre o corpo e de padrões de hábitos cotidianos para superação dos problemas psicossociais.

Sendo assim, a cirurgia pode ser vista como uma oportunidade para melhorar a vida dos indivíduos obesos, mas é preciso que se deixe claro que esse tratamento, a princípio bastante fácil, exigirá, por longo tempo, muito empenho da pessoa operada, de acompanhamento médico periódico e da colaboração direta de pessoas amigas e de profissionais dedicados à prestação de cuidados de enfermagem, de psicologia e de nutrição específicas. Para tanto, é necessário fazer valer a inclusão da família e de amigos na fase preparatória, conforme prescrito nos protocolos, e ainda garantir que a atenção dos profissionais contemple a compreensão da subjetividade de cada caso e dos demais fatores que reforçam a complexidade da obesidade como um fenômeno particular e que exige abordagem interdisciplinar.

Por princípio ético, referente à finalidade da pesquisa, entende-se que seja importante apontar recomendações, com vistas a contribuir para o aperfeiçoamento das práticas profissionais e das políticas públicas de educação e saúde no país:

- Garantir que as informações quanto aos "ganhos e perdas" do processo cirúrgico sejam discutidas nos serviços de saúde, com bastante antecedência, pela ética e responsabilidade compartilhada, com os indivíduos que pretendam fazer este tipo de tratamento. Desenvolver espaços para a relação cirurgião-paciente, em particular, para garantir que os critérios rigorosos para a indicação da cirurgia bariátrica sejam discutidos de maneira a evidenciar os riscos e as consequências desse tratamento e ainda as possibilidades reais de atenção integral à sua saúde no decorrer de todo o processo;

- Antes de supervalorizar o tratamento cirúrgico, é necessário que equipes de saúde estejam preparadas para empreender esforços para desenvolver o cuidado integral, de maneira a contribuir para a prevenção e o combate da obesidade, evitando-se, assim, a atitude extrema de indicar tratamento tão invasivo;

- Perceber a criação de estratégias e condutas relacionadas ao controle da obesidade como um dos grandes desafios dos profissionais e progra- mas de saúde. Em nível básico de atenção, é necessário avançar para a proteção específica, com diagnóstico precoce dos casos de obesidade de difícil manejo;

- Manter atualização sobre o estado da arte das experiências relativas à cirurgia bariátrica. Buscar informações atualizadas sobre as contraindicações e as ocorrências de complicações físicas e psíquicas. Estas, se diagnosticadas a tempo junto à pessoa operada, possibilitam atenção mais focada e encaminhamentos para especialistas, evitando maiores danos à saúde, sofrimentos psicosociais e a morte prematura. Orienta-se consultar a ABESO (Associação Brasileira para o Estudo da Obesidade e da Síndrome Metabólica) e, sistematicamente, o site dos SUS especializado nesse âmbito - http://www.gastroplastia. net/sus.html;

- Promover encontros de grupo para a discussão sobre os protocolos existentes para a atenção pré e pós-operatória, integrando diferentes profissionais da área da saúde, cuidadores de domicílio e indivíduos que tenham se submetido à cirurgia.

Ressalta-se a importância de os profissionais inseridos no cenário da saúde coletiva investirem mais no seu papel de mediador, de educador em saúde, reconhecendo a necessidade de um envolvimento maior nas ações de promoção da saúde nos contextos família e escola com vistas à prevenção da obesidade. O caminho para estas estratégias exige a atuação interdisciplinar, pela qual a atenção ao processo saúde-doença das pessoas seja permeada pelo olhar integrado e sincronizado com os hábitos socioculturais da população. Sugere-se a apreciação da Carta Europeia de Luta contra a Obesidade, resultante da Conferência Ministerial da Organização Mundial de Saúde Europeia sobre a Luta contra a Obesidade (Istambul, 2006) $)^{25}$.

Às instituições formadoras de profissionais, ressalta-se o quão importante é seu papel para a mudança da realidade da obesidade no país. Nesse sentido, atendendo à perspectiva interdisciplinar e intersetorial, é preciso dar reforço a essa temática em disciplinas e pesquisas nos cursos das áreas da saúde, da educação e das ciências humanas e sociais aplicadas que reconheçam a importância da saúde coletiva num caminho focado no cuidado de promoção, capaz de contribuir para remodelar as fontes midiáticas e as práticas de saúde, particularmente aquelas relacionadas à alimentação e aos demais hábitos e estilos de vida promotores da obesidade. 


\section{Colaboradores}

LF Marcelino trabalhou na concepção do estudo, metodologia, coleta dos dados, análise e in- terpretação dos dados e na elaboração do artigo; e MZ Patrício trabalhou na concepção do estudo, metodologia, análise e na interpretação dos dados e na elaboração do artigo.

\section{Referências}

1. Organização Mundial de Saúde (OMS). Doenças não-transmissíveis: estratégia regional para 2000-2010. [acessado 2005 out 21]. Disponível em: www.afro. who.int/textonly/press/portuguese/2000/rc/rc5006. html

2. Castro C. Ministros da Saúde das Américas se comprometem a criar políticas públicas para reduzir a obesidade no continente. São Paulo: Associação Brasileira para o Estudo da Obesidade - ABESO; 2011. [acessado 2011 mar 6]. Disponível em: http://www. abeso.org.br/.

3. Organização Pan-Americana da Saúde (OPAS). Doenças crônico-degenerativas e obesidade: estratégia mundial sobre alimentação saudável, atividade física e saúde. Brasília: Organização Pan-Americana da Saúde (OPAS); 2003.

4. Brasil. Ministério da Saúde (MS). A vigilância, o controle e a prevenção das doenças crônicas não-transmissíveis: DCNT no contexto do Sistema Único de Saúde brasileiro. Brasília: Organização Pan-Americana da Saúde (OPAS), Organização Mundial de Saúde (OMS); 2005.

5. Segal A, Fandiño J. Indicações e contra indicações para a realização das operações Bariátricas. Rev. Bras. Psiq. 2002; 24(Supl. 3):68-71.

6. Garrido Júnior AB, Ferraz EM, Barroso FL, Marchesini JB, Szëgo T. Cirurgia da obesidade. São Paulo: Atheneu; 2002.

7. Sabiston Júnior DC. Tratado de cirurgia: as bases biológicas da prática cirúrgica moderna. Rio de Janeiro: Guanabara Koogan; 1999.

8. Suplicy H. Avaliando a obesidade a sua comorbidade. Serviço de Endocrinologia e Metabologia do Paraná/Hospital de Clínicas da UFP; 2005. [acessado 2005 set 23]. Disponível em: http://www.endocrino. org.br/artigos_obesidade_001.php.

9. Repetto G, Rizzolli J, Bonatto C. Prevalência, riscos e soluções na obesidade e sobrepeso: Here, There, and Everywhere. Arq Bras Endocrinol Metab 2003; 47(6):633-635.

10. Carvalho PS, Moreira CLCB, Barelli MC, Oliveira FH, Guzzo MF, Miguel GPS, Zandonade S. Cirurgia bariátrica cura síndrome metabólica? Arq Bras Endocrinol Metab 2007; 51(1):79-85.

11. Brasil. Ministério da Saúde (MS). Obesidade: SUS realiza três novos tipos de cirurgia para redução de estômago. [acessado 2011 fev 24]. Disponível em: http://portal.saude.gov.br/saude

12. Ministério da Saúde (MS). Cirurgia Bariátrica no Tratamento da Obesidade Mórbida. Boletim Brasileiro de Avaliação de Tecnologias em Saúde 2008; III(5). [acessado 2011 mar 4]. Disponível em: http:// 200.214.130.94/ rebrats/publicacoes/Brats05.pdf.
13. Sociedade Brasileira de Cirurgia Bariátrica e Metabólica (SBCBM). [acessado 2011 fev 24]. Disponível em: http://www.sbcb.org.br/

14. Brasil. Secretaria de Vigilância em Saúde (SVS). Saúde Brasil 2004: uma análise da situação de saúde. Brasília, DF: Secretaria de Vigilância em Saúde (SVS); 2004.

15. Brasil. Ministério da Saúde (MS). Gastroplastia é a solução para a obesidade mórbida. Brasília, DF; 2004. [acessado 2004 maio 5]. Disponível em: http:// portal.saude.gov.br/saude.

16. Minayo MCS. O desafio do conhecimento: pesquisa qualitativa em saúde. 6a ed. São Paulo: Hucitec; 1999.

17. Patrício ZM. O processo ético e estético de pesquisar: um movimento qualitativo transformando conhecimentos e a qualidade de vida individual-coletiva. Florianópolis: UFSC; 2004.

18. Appolinário JC. Obesidade e psicopatologia. In: Halpern A, Godoy MAF, Suplicy HL, Mancini MC, Zanella MT, organizadores. Obesidade. São Paulo: Lemos Editorial; 1998. p. 217-227.

19. Brander N. Autoestima e os seus seis pilares. São Paulo: Saraiva; 2000.

20. Puttini S. Obesidade: tratamento cirúrgico. Rev. Nutrição 2004; 4(17).

21. Loss AB, Souza AAP, Pitombo CA, Milcent M, Madureira FAV. Avaliação da síndrome de dumping em pacientes obesos mórbidos submetidos à operação de bypass gástrico com reconstrução em Y de Roux. Rev Col Bras Cir 2009; 36(5):413-419.

22. Carvalho MCVS, Luz MT. Simbolismo sobre "natural" na alimentação. Cien Saude Colet 2011; 16(1):147-154.

23. Silva S, Maia AC. Experiências adversas na infância e tentativas de suicídio em adultos com obesidade mórbida. Rev. psiquiatr. RS 2010; 32(3):69-72

24. Magdaleno Júnior R, Chaim EA, Turato ER. Características psicológicas de pacientes submetidos a cirurgia bariátrica. Rev. psiquiatr. RS 2009; 31(1):73-78.

25. Plataforma Contra a Obesidade. Carta Europeia de Luta contra a Obesidade. Conferência Ministerial da Organização Mundial de Saúde Europeia sobre a Luta contra a Obesidade (Istambul, 2006). [acessado 2011 mar 5]. Disponível em: http://www. plataformacontraaobesidade.dgs.pt/PresentationLayer/conteudo.aspx?menuid=113http://www.fipa. pt/ userfiles/file/Carta\%20Euro

Artigo apresentado em 25/04/2008

Aprovado em 16/12/2008

Versão final apresentada em 16/03/2011 